\title{
Effect of Traffic Pattern on Packet Delivery Ratio in Reactive Routing Protocol of Manet
}

\author{
Patil V.P. \\ Smt. Indira Gandhi College of Engineering, New Mumbai, India.
}

\begin{abstract}
An ad hoc network is a collection of mobile nodes that dynamically form a temporary network. Routing in a MANET is challenging because of the dynamic topology and the lack of an existing fixed infrastructure. In such a scenario a mobile host can act as both a host and a router forwarding packets for other mobile nodes in the network. Routing protocols used in mobile ad hoc networks (MANET) must adapt to frequent or continual changes of topology, while simultaneously limiting the impact of tracking these changes on wireless resources. In his paper investigation has been done on the effect of change in number of nodes, node speed and pause time on packet delivery ratio (PDR) of MANET reactive routing protocols. Here, it has been analyzed and compared the performance of MANET routing protocols AODV and DSR based on both CBR and $T C P$ based traffic patterns. The NS-2 simulator is used for performing various simulations and used awk scripts for analyzing the results. AODV in the simulation experiment shows the overall best performance. It has an improvement over DSR.
\end{abstract}

Keywords: Aodv, Cbr, Dsr, Manet, Ns2, Tcp,

\section{Introduction}

A MANET [1,2] consists of a number of mobile devices that come together to form a network as needed, without any support from any existing Internet infrastructure or any other kind of fixed stations. Formally, a MANET can be defined as an autonomous system of nodes or MSs also serving as routers connected by wireless links, the union of which forms a communication network modeled in the form of an arbitrary communication graph. In such environment, Neighbor nodes communicate directly with each other's while communication between non-neighbor nodes performed via the intermediate nodes which act as routers. As the network topology changes frequently because of node mobility and power limitations, efficient routing protocols are necessary to organize and maintain communication between the nodes.

MANETs have several salient characteristics: i) Dynamic topologies ii) Bandwidth constrained, variable capacity links, iii) Energy-constrained operation and limited physical security etc. Therefore the routing protocols used in ordinary wired networks are not well suited for this kind of dynamic environment. In this paper an effort has been done to evaluate the routing performance of AODV and DSR using Network Simulator NS2 and results have been analyzed.

The rest of the paper is organized as follows: Section II presents the mobile ad hoc routing protocols. Section III provides an overview and general comparison of the routing protocols used in the study. The related work is described in section IV. The simulation environment and performance metrics are described in Section $\mathrm{V}$ and then the results are presented in Section VI. Section VII concludes the paper.

\section{Routing Protocols}

Routing protocols for Mobile ad hoc networks can be broadly classified into two main categories: $\emptyset$ Proactive or table-driven routing protocols

$\varnothing$ Reactive or on-demand routing protocols.

\subsection{On-Demand Routing Protocols (Reactive)}

Reactive routing protocols [3], [4] try to utilize network bandwidth by creating routes only when desired by the source node. Once a route has been established, it is maintained by some route maintenance mechanism as long as it is needed by the source node. When a source node needs to send data packets to some destination, it checks its route table to determine whether it has a valid route. If no route exists, it performs a route discovery procedure to find a path to the destination. Hence, route discovery becomes on-demand. These routing approaches are well known as Reactive routing. Examples of reactive (also called on-demand) ad hoc network routing protocols include ad hoc on-demand distance vector (AODV), temporally ordered routing algorithm (TORA), dynamic source routing (DSR)[5]. 


\subsection{Table Driven Routing Protocols (Proactive)}

In proactive or table-driven routing protocols, each node continuously maintains up-to-date routes to every other node in the network. Routing information is periodically transmitted throughout the network in order to maintain routing table consistency. Thus, if a route has already existed before traffic arrives, transmission occurs without delay. Otherwise, traffic packets should wait in queue until the node receives routing information corresponding to its destination. However, for highly dynamic network topology, the proactive schemes require a significant amount of resources to keep routing information up-to-date and reliable. Certain proactive routing protocols are Destination-Sequenced Distance Vector (DSDV) [9], Wireless Routing Protocol (WRP) [10, 11], Global State Routing (GSR) [11] and Cluster head Gateway Switch Routing (CGSR) [11].

\section{Overview Of Aodv, Dsr Routing Protocols And Traffic Pattern Types}

Every routing protocol has its own merits and demerits, none of them can be claimed as absolutely better than others. In this paper the two reactive routing protocols - AODV, DSDV has been selected for evaluation.

\subsection{Ad hoc On-demand Distance Vector Routing (AODV)}

Ad-hoc On-demand distance vector (AODV) $[12,13]$ is another variant of classical distance vector routing algorithm, a confluence of both DSDV [9] and DSR [14]. It shares DSR's on-demand characteristics hence discovers routes whenever it is needed via a similar route discovery process. However, AODV adopts traditional routing tables; one entry per destination which is in contrast to DSR that maintains multiple route cache entries for each destination. The initial design of AODV is undertaken after the experience with DSDV routing algorithm. Like DSDV, AODV provides loop free routes while repairing link breakages but unlike DSDV, it doesn't require global periodic routing advertisements. AODV also has other significant features. Whenever a route is available from source to destination, it does not add any overhead to the packets. However, route discovery process is only initiated when routes are not used and/or they expired and consequently discarded. This strategy reduces the effects of stale routes as well as the need for route maintenance for unused routes. Another distinguishing feature of AODV is the ability to provide unicast, multicast and broadcast communication. AODV uses a broadcast route discovery algorithm and then the unicast route reply massage.

\subsection{Dynamic source routing (DSR)}

Dynamic source routing (DSR) [5] is based on source routing where the source specifies the complete path to the destination in the packet header. All intermediary nodes along the path simply forwards the packet to the next node as specified in the packet header $[5,6]$. This means that intermediate nodes only need to keep track of their neighboring nodes to forward data packets. The source on the other hand, needs to know the complete hop sequence to the destination. This eliminates the need for maintaining latest routing information by the intermediate nodes as in DSDV. In DSR, all nodes in a network cache the latest routing information. When more than one route to the destination is found, the nodes cache all the route information so that in case of a route failure, the source node can look up their cache for other possible routes to the destination. If an alternative route is found, the source node uses that route; else the source node will initiate route discovery operations to determine possible routes to the destination. During route discovery operation, the source node floods the network with query packets. Only the destination or a node which already knows the route to destination can reply to it, hence avoiding the further propagation of query packets from it. If a broken link is detected by a node, it sends route error messages to the source node. The source node on receiving error messages will initiate route discovery operations. Unlike DSDV, there are no periodically triggered route updates.

\subsection{Traffic type:}

There are two types of traffic patterns used in this paper a) TCP and b) UDP (CBR)

\section{a)Transmission Control Protocol (TCP)}

It is often referred to as TCP/IP due to the importance of this protocol in the Internet Protocol Suite. TCP operates at a higher level, concerned only with the two end systems, (e.g. between web browser and a web server). TCP provides reliable, sequential delivery of a stream of data from one program on one computer to another program on another computer. Common uses of TCP are e-mailing support, file transfer, Web applications. Among its features, TCP controls message size, the rate at which messages are exchanged, and network traffic congestion. As for IP, it handles lower-level transmissions from computer to computer as a message transferred across the Internet.

\section{b)User Datagram Protocol (UDP)}

It is part of the base protocols of the Internet Protocol Suite. Programs on networked computers can send short messages sometimes called datagrams. UDP does not guarantee any reliability (it happens that 
Effect of Traffic Pattern on Packet Delivery Ratio in Reactive Routing Protocol of MANET

datagram may be out of order, duplicated, or goes missing without any notice). The fact that no checking whether all packets are actually delivered is made, UDP proves to be faster and more efficient, for applications that do not need guaranteed delivery. UDP find its uses in situations like Time-sensitive applications where the problems due to delayed packets are avoided and It is also useful for servers that answer small queries from huge numbers of clients. UDP supports packet broadcast (conveys to all on local network) and multicasting (conveys to all subscribers).

\section{Related Work}

Several researchers have done the qualitative and quantitative analysis of Ad-hoc Routing Protocols by means of different performance metrics. They have used different simulators for this purpose. Broch et al. [19], conducted experiments with DSDV, TORA, DSR and AODV. They used a constant network size of 50 nodes, 10 to 30 traffic sources, seven different pause times and various movement patterns. Packet delivery fraction (PDF), number of routing packets and distribution of path lengths were used as performance metrics. They extended $n s-2$ discrete event simulator [20], developed by the University of California at Berkeley and the VINT project [21], to correctly model the MAC and physical-layer behavior of the IEEE 802.11 wireless LAN standard. Ehsan and Uzmi [22], presented the performance comparison of DSDV, AODV, DSR and TORA based on simulations performed using network simulator-2. Three metrics: normalized routing overhead, packet delivery fraction and average end to end delay, were used to measure performance. Karthikeyan et al. [23] studied the Reactive protocols, DSR and AODV as well as a Proactive Protocol, DSDV and their characteristics with respect to different mobility were analyzed based on packet delivery fraction, routing load, end to-end delay, number of packets dropped, throughput and jitter using Network Simulator (ns-2). However, in all the cases, only CBR sources were used for generating traffic.

\section{Proposed Methodology And Performance Metrics}

\subsection{Problem formulation:}

The research in this MANET area has continued with prominent studies on routing protocols such as Ad hoc On-demand Distance Vector (AODV), Destination-Sequenced Distance-Vector Routing protocol, (DSDV) and Dynamic Source Routing Protocol (DSR). Several studies have been done on the performance evaluation of routing protocols based on CBR traffic pattern using different evaluation methods. Different methods and simulation environments give different results and consequently, there is need to broaden the spectrum to account for effects not taken into consideration in a particular environment. It is observed that most of the research work is based on CBR traffic pattern whereas most of the traffic approximately $95 \%$ on the Internet carries TCP. It is desirable to study and investigate the Performance of different MANET routing protocols under both CBR and TCP traffic patterns. In this paper, evaluation has been done for the performance of Reactive protocols i.e Ad hoc on demand distance vector routing (AODV) and dynamic source routing (DSR) of mobile ad-hoc network routing protocols for both CBR and TCP traffic patterns. The performance of these routing protocols is evaluated with respect to effect on packet delivery ratio due to variation in node density, pause time and node speed.

\subsection{Performance metrics:}

Design and performance analysis of routing protocols used for mobile ad hoc network (MANET) is currently an active area of research. To judge the merit of a routing protocol, one needs metrics both- qualitative and quantitative- with which to measure its suitability and performance. Specifically, this paper evaluates the performance comparison of AODV and DSR reactive routing protocols . The Packet Delivery Ratio (PDR) performance metrics is used to compare the performance of these routing protocols in the simulation by varying node density, pause time ,node speed and for he two traffic patterns TCP and UDP(CBR).

Packet delivery ratio is calculated by dividing the number of packets received by the destination through the number of packets originated by the application layer of the source. It specifies the Packet loss rate, which limits the maximum throughput of the network. The better the delivery ratio, the more complete and correct is the routing protocol.

\section{1 simulations}

\section{Simulation, Results And Performance Analysis}

In this paper, two different scenarios have been taken. In the first scenario, traffic pattern is taken as TCP and no. of nodes, pause time and node speed have been varied and performance comparison has been made between AODV and DSR protocols. In the second scenario, traffic pattern is taken as UDP (CBR) and no. of nodes, pause time and node speed have been varied and a performance comparison has been made between AODV and DSR protocols. Identical mobility pattern are used across protocols to gather fair results.

The source destination pairs are spread randomly over the network. The mobility model uses 'random waypoint model' [24] in a rectangular filed of $1000 \mathrm{~m} \mathrm{x} \mathrm{700m} \mathrm{with} 25$ nodes. During the simulation, each node 
starts its journey from a random spot to a random chosen destination. Once the destination is reached, the node takes a rest period of time in second and another random destination is chosen after that pause time. This process repeats throughout the simulation, causing continuous changes in the topology of the underlying network. Different network scenario for different number of nodes and pause times are generated. The model parameters that have been used in the following experiments are summarized in Table 1and 2.

\subsubsection{Test Scenario 1}

In the first scenario, the simulation based on TCP traffic pattern is selected. Parameters of this scenario are summarized in table 1 . Here, TCP sources are used which use flow control and retransmission feature.

Table 1: Simulation parameters for test case of TCP

\begin{tabular}{|l|l|}
\hline PARAMETERS & VALUES \\
\hline No. Of Nodes & $10,20,30,40,50$ \\
\hline Simulation Time & 500 Seconds \\
\hline Environment Size & $1000 \times 700$ \\
\hline Simulation Speed & $2,5,8,10,12 \mathrm{~m} / \mathrm{sec}$ \\
\hline Pause Time & $0,50,100,150,200$ \\
\hline Packet Size & 512 bytes \\
\hline Routing protocols & AODV,DSR \\
\hline Traffic Type & TCP \\
\hline Simulator type & NS2 -2.34 \\
\hline Mobility model & Random way point \\
\hline
\end{tabular}

\subsubsection{Test case Scenario 2}

In the second scenario, the simulation based on CBR traffic pattern has been chosen. Parameters of this scenario are summarized in table 2. CBR sources are used that started at different times to get a general view of how routing protocol behaves.

Table 2: Simulation parameters for test case of UDP(CBR)

\begin{tabular}{|l|l|}
\hline PARAMETERS & VALUES \\
\hline No. Of Nodes & $10,20,30,40,50$ \\
\hline Simulation Time & 500 Seconds \\
\hline Environment Size & $1000 x 700$ \\
\hline Simulation Speed & $2,5,8,10,12 \mathrm{~m} / \mathrm{sec}$ \\
\hline Pause Time & $0,50,100,150,200$ \\
\hline Packet Size & 512 bytes \\
\hline Routing protocols & AODV,DSR \\
\hline Traffic Type & CBR \\
\hline Simulator type & NS2 -2.34 \\
\hline Mobility model & Random way point \\
\hline
\end{tabular}

\subsection{Results and performance comparison}

Performance of AODV and DSR protocols is evaluated under both CBR and TCP traffic pattern. Extensive Simulation is done by using NS-2.

The simulation results are shown in the form of line graphs. Graphs show comparison between the two protocols by varying different numbers of sources on the basis of the Packet delivery ratio as metrics and by varying node density, node speed, and pause time.

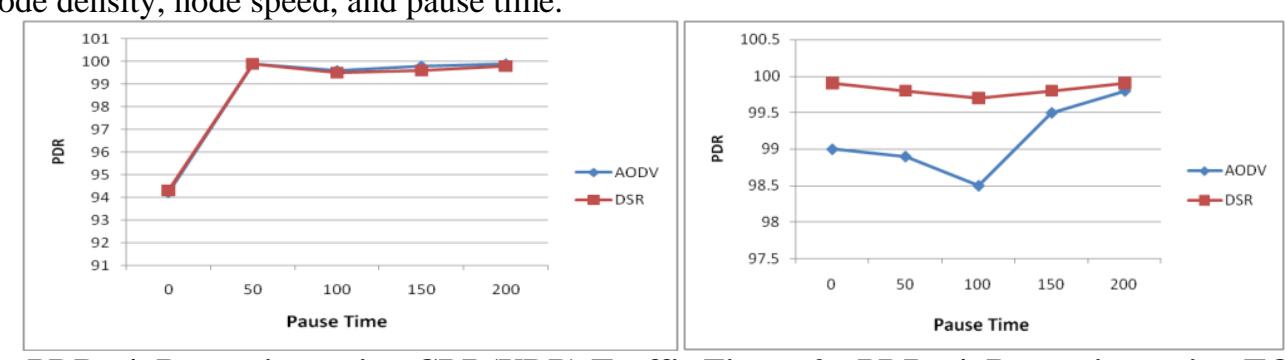

Figure 1 : PDR v/s Pause time using CBR(UDP) Traffic Figure 2 : PDR v/s Pause time using TCP Traffic 


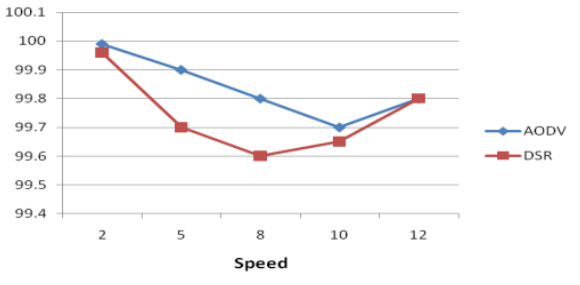

Figure 3 : PDR v/s Speed using CBR(UDP) Traffic
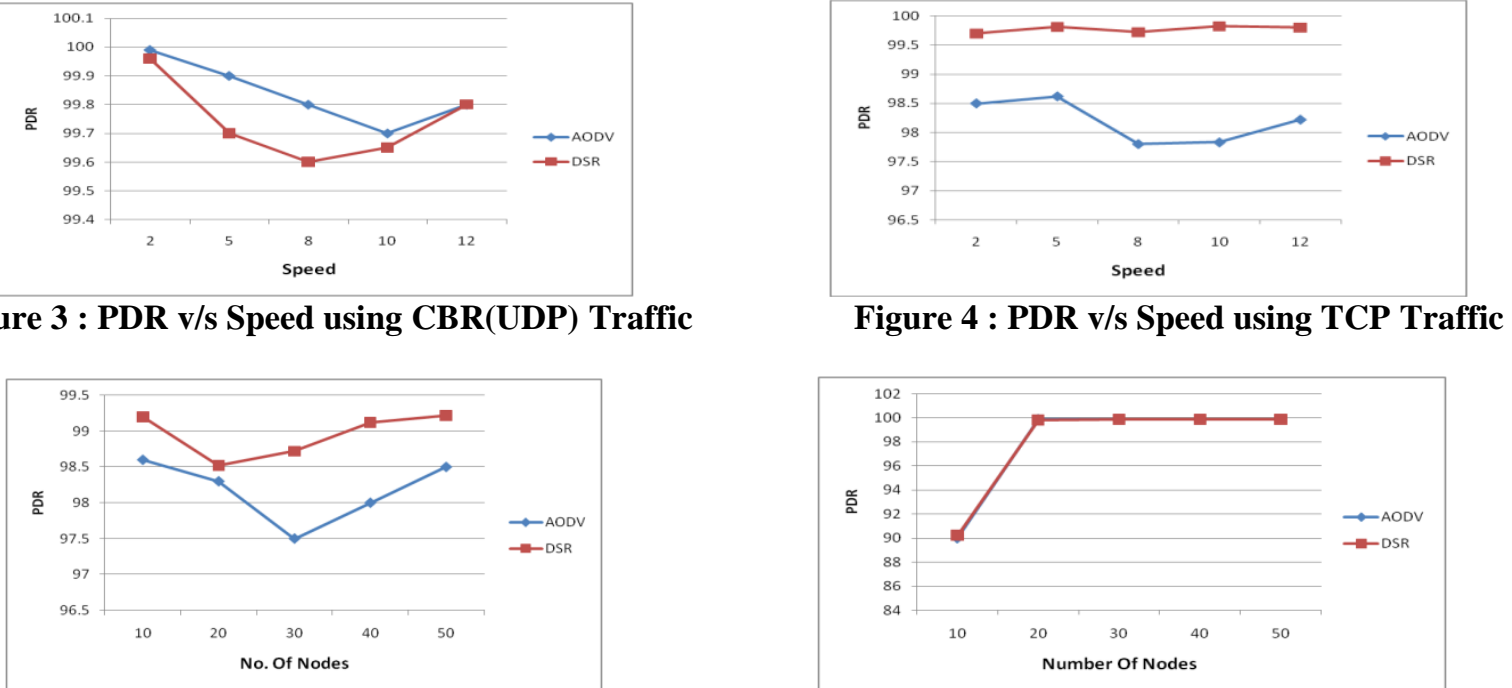

Figure -5: PDR v/s No. of Nodes for TCP Traffic

Traffic

Figure 4 : PDR v/s Speed using TCP Traffic

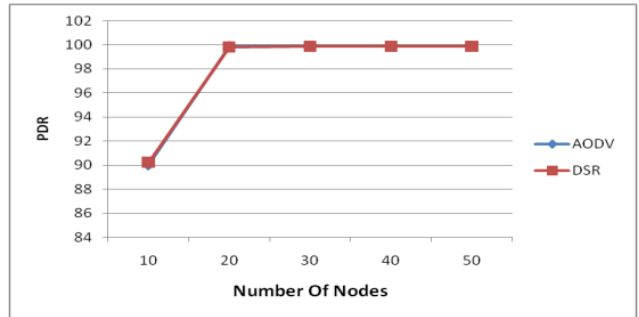

Figure- 6:PDR v/s No. of Nodes for CBR

Figure-1 shows a comparison between the routing protocols on the basis of packet delivery ratio as a function of pause time and using CBR (UDP) traffic source with both routing protocols AODV and DSR. It reflects the completeness and accuracy of the routing protocols. It shows very clear that the performance of AODV and DSR almost same. Pause time of 0 denotes maximum movement and 200 denote least movement. It shows at the 0 pause time PDR is very less, its around $94 \%$ in both protocols. When pause time is increased the PDR will be increased but after a particular pause time the PDR will be almost stable.

Figure-2 shows the comparison between AODV and DSR using on the basis of PDR as a function basic of pause time using TCP traffic source. In this scenario all the PDR outputs above 99\%, But it shows DSR's PDR is little bit high compared to AODV.

Figure-3 shows the comparison between AODV and DSR using on the basis of PDR as a function basic of Speed using CBR (UDP) traffic source. In this scenario all the PDR outputs above 99\%, but it shows AODV's PDR better compare to DSR at all the speed points.

Figure-4 shows the comparison between AODV and DSR using on the basis of PDR as a function basic of Speed using TCP traffic source. In this scenario all the PDR outputs above 98\%, but it shows DSR's PDR better compare to AODV at all the speed points.

Figure -5 Packet Delivery Ratio v/s No. of Nodes for TCP Traffic: A simulation result of Figure-5 shows that varying no. of nodes: i) Packet delivery ratio for both protocols with varying no. of nodes is stable. ii) DSR has slightly more packet delivery ratio than AODV.

Figure- 6 Packet Delivery Ratio v/s No. of Nodes for CBR Traffic: For scenario-2 graph generated in Fig. 6 shows that Packet Delivery ratio of two protocols DSR, AODV is similar.

\section{Conclusion}

This study was conducted to evaluate the performance of two MANET protocols i.e. AODV, and DSR based on CMU's generated TCP \& CBR traffic. These routing protocols were compared in terms of Packet delivery ratio, when subjected to change in no. of nodes \& traffic type, Pause time and node speed. In this paper, evaluation of the routing protocols, unlike previous works of similar nature, brings out the effect of routing protocols on the TCP performance. Through experiments, first the essential differences between TCP and UDP traffic and therefore need to consider TCP traffic for routing protocol performance evaluation. Based on these experiments performance metrics i.e. Packet delivery ratio was measured for quantifying the performance of the protocols.

AODV in the simulation experiment shows the overall best performance. It has an improvement over DSR. It has been further observed that due to the dynamically changing topology and infrastructure less, decentralized characteristics, security and power awareness is hard to achieve in mobile ad hoc networks. Hence, security and power awareness mechanisms should be built-in features for all sorts of applications based on ad hoc network. Performance can also be analyzed for other parameters like Jitter, Routing Overhead, throughput and average end to end delay. By evaluating the performance of these protocols new protocols can be implemented or changes can be suggested in the earlier protocols to improve the performance. 


\section{References}

[1] C. E. Perkins and E. M. Royer. "The ad hoc on-demand distance vector protocol," Ad Hoc Networking, Addison-Wesley, pp. 173219,2001

[2] D. B. Johnson, D. A. Maltz, and J. Broch, "DSR: the dynamic source routing protocol for multi-hop wireless ad hoc networks," in Ad Hoc Networking, Addison-Wesley, 2001, pp. 139-172.

[3] Johnson, D. B., Maltz, D. A., Hu, Y. C. and Jetcheva, J. G. 2002. "The dynamic source routing protocol for mobile ad hoc networks (DSR)". Draft-ietf-manet-dsr-07.txt.

[4] Perkins, C. E., Royer, E. M. and Das, S. R. 1999. "Ad Hoc On-Demand Distance Vector Routing”. IETF Internet Draft. http://www.ietf.org/internet-drafts/draft-ietf-manetaodv-03.txt.

[5] Anuj K. Gupta, Dr. Harsh Sadawarti, Dr. Anil K. Verma, "Performance analysis of AODV, DSR and TORA Routing Protocols", International Journal of Engineering \& Technology (IJET), ISSN: 1793-8236, Article No. 125, Vol.2 No.2, April 2010, pp. - 226231.

[6] Narendran Sivakumar, Satish Kumar Jaiswal, "Comparison of DYMO protocol with respect to various quantitative performance metrics", ircse 2009 .

[7] NS-2, The ns Manual (formally known as NS Documentation) available at http://www.isi.edu/nsnam/ns/doc.NAM

[8] http://www.isi.edu/nsnam/nam/

[9] C. E. Perkins and P. Bhagwat, "Highly Dynamic Destination- Sequenced Distance-Vector Routing (DSDV) for Mobile Computers," SIGCOMM, London, UK, August 1994, pp. 234-244.

[10] Vincent D. Park and M.Scott Corson. "A highly adaptive distributed routing algorithm for mobile wireless networks".In Proceedings of INFOCOM 1997, 1997.

[11] Saleh Ali K.AI-Omari \& Putra Sumari "An overview of mobile adhoc networks for the existing protocols and applications"Internattional Journal on Applications of Graph theory in wireless adhoc networks and sensor networks (Graph-Hoc), Vol2 No1 March 2010.

[12] C. E. Perkins and E. M. Royer, "Ad Hoc On-demand Distance Vector Routing," In Proceedings of the 2nd IEEE Workshop on Mobile Computing Systems and Applications, New Orleans, LA, February 1999, pp. 90-100.

[13] C. Perkins, "Ad hoc On demand Distance Vector (AODV) routing", IETF Internet draft (1997),http://www.ietf.org/internetdrafts/draftietf- manet-aodv-00.txt.

[14] D. B. Johnson and D. A. Maltz, "Dynamic Source Routing in Ad-Hoc Ad hoc Networks," Mobile Computing, ed. T. Imielinski and H. Korth, Kluwer Academic Publishers, 1996, pp. 153-181.

[15] Dyer T. D., Boppana R. V., “ A Comparison of TCP performance over three routing protocols for mobile adhoc networks “, ACM Symposium on Mobile Adhoc Networking \& Computing (Mobihoc), October 2001.

[16] Suresh Kumar, R K Rathy and Diwakar Pandey, "Traffic pattern based performance comparison of two reactive routing protocols for ad hoc networks using NS2”, (C) 2009 IEEE.

[17] Vikas Singla, Rakesh Singla and Ajay Kumar,"Performance Evaluation and Simulation of Mobile Ad-hoc Network Routing Protocols", International Journal of Engineering and Information Technology, Volume 1 No. 1 October 2009.

[18] Yogesh Chaba, Yudhvir Singh, Manish Joon, "Simulation Based Performance Analysis of On-Demand Routing Protocols in MANETs," Second International Conference on Computer Modeling and Simulation, 2010.

[19] J. Broch, D. A. Maltz, D. B. Johnson, Y. C. Hu and J. Jetcheva, "A Performance Comparison of Multi-Hop Wireless Ad-hoc Network Routing Protocols," in Proceedings of the 4th Annual ACM/IEEE International Conference on Mobile Computing and Networking (MOBICOM'98), October 1998, pp. 85-97.

[20] Information Sciences Institute, "ns-2 network simulator," Software Package, 2003. [Online]. Available: http://www.isi.edu/nsnam/ns/

[21] “The VINT Project," USC/ISI, Xerox PARC, LBNL and UC Berkeley,1997. [Online].Available: http://www.isi.edu/nsnam/vint/

[22] Humaira Ehsan and Zartash Afzal Uzmi, "Performance Comparison of Ad-hoc Wireless Network Routing Protocols", IEEE Transactions, 2004.

[23] N. Karthikeyan, V. Palanisamy and K. Duraiswamy, "A Performance Evaluation Of Proactive And Reactive Protocols Using ns-2 Simulation”, International J. of Engg. Research \& Indu. Appls. (IJERIA).ISSN 0974-1518, Vol.2, No.II (2009), pp 309-326.

[24] Mohd Izuan Mohd Saad "Performance Analysis of Random-Based Mobility Models in MANET Routing Protocol" European Journal of Scientific Research ISSN 1450-216X Vol.32 No.4 (2009), pp.444-454 @ EuroJournals Publishing, Inc. 2009. 